\title{
Recomendações para o sono seguro do lactente
}

\author{
Recommendations for safe infant sleep \\ Recomendaciones para un sueño infantil seguro
}

\section{Resumo}

Objetivo: Identificar e orientar, na literatura o posicionamento redutor do acometimento da Síndrome da Morte Súbita do Lactente (SMSL). Metodologia: trata-se de revisão integrativa de literatura, onde foi realizado um levantamento no nas bases de dados: Scientific Electronic Library Online (Scielo), CINAHL, Science Direct, Medline, Literatura Latino-Americana e do Caribe em Ciências da Saúde (LILACS), MedCarib, PAHO, SCOPUS, Wholis e Embase. Utilizando os descritores "morte súbita", "neonato", "recém-nascido", "decúbito dorsal", "decúbito ventral" com o uso dos operadores booleanos "and" e "or”. Resultados: Após a aplicação dos critérios de inclusão e exclusão, 14 artigos foram qualificados onde 12 orientaram o decúbito dorsal como prevenção para SMSL. Demonstra-se que os principais mecanismos de lesão associados a SMSL são: o uso de colecho/co-sleeping (21\%), exposição ao tabaco (18\%), objetos macios na cama (18\%), decúbito lateral (15\%), uso de drogas e alccol durante a gestação (12\%) e uso de cobertores (12\%). Discussão: Os achados demonstraram a predominância do decúbito dorsal como medida de recomendação e orientação para prevenção da SMSL. Conclusões: A SMSL é possivelmente subnotificada e associada a outros diagnósticos, recomenda-se para os profissionais de saúde insistirem e incentivarem as mães a colocar o recém-nascido a dormir em decúbito dorsal, a fim de prevenir a SMSL.

Palavras-chave: Morte súbita; Recém-nascido; Decúbito dorsal.

\begin{abstract}
Objective: To identify and guide, in the literature, the position that reduces the involvement of Sudden Infant Death Syndrome (SIDS). Methodology: this is an integrative literature review, where a survey was carried out in the following databases: Scientific Electronic Library Online (Scielo), CINAHL, Science Direct, Medline, Latin American and Caribbean Health Sciences (LILACS) ), MedCarib, PAHO, SCOPUS, Wholis and Embase. Using the descriptors "sudden death", "newborn", "newborn", "dornal decubitus", "ventral decubitus" with the use of the Boolean operators "and" and "or". Results: After applying the inclusion and exclusion criteria, 14 articles were qualified where 12 guided the supine position as prevention for SIDS. The main mechanisms of
\end{abstract}


injury associated with SIDS are shown to be: the use of mattresses/co-sleeping (21\%), exposure to tobacco (18\%), soft objects in bed (18\%), lateral decubitus (15\%), use of drugs and alcohol during pregnancy (12\%) and use of blankets (12\%). Discussion: The findings demonstrated the predominance of the supine position as a measure of recommendation and guidance for SIDS prevention. Conclusions: SIDS is possibly underreported and associated with other diagnoses, it is recommended that health professionals insist and encourage mothers to put the newborn to sleep in the supine position, in order to prevent SIDS.

Keywords: Sudden death; Newborn; Supine.

\section{Resumen}

Objetivo: Identificar y orientar, en la literatura, la posición que reduce la participación del Síndrome de Muerte Súbita del Lactante (SMSL). Metodología: se trata de una revisión integradora de la literatura, donde se realizó una encuesta en las siguientes bases de datos: Scientific Electronic Library Online (Scielo), CINAHL, Science Direct, Medline, Latin American and Caribbean Health Sciences (LILACS), MedCarib, OPS, SCOPUS, Wholis y Embase. Utilizando los descriptores "muerte súbita", "recién nacido", "recién nacido", "decúbito dornal”, "decúbito ventral" con el uso de los operadores booleanos "and" y "or". Resultados: Después de aplicar los criterios de inclusión y exclusión, se calificaron 14 artículos donde 12 orientaron la posición supina como prevención del SMSL. Se muestra que los principales mecanismos de lesión asociados al SMSL son: uso de colchones / colecho (21\%), exposición al tabaco (18\%), objetos blandos en la cama (18\%), decúbito lateral (15\%), uso de drogas y alcohol durante el embarazo (12\%) y uso de mantas (12\%). Discusión: Los hallazgos demostraron el predominio de la posición supina como medida de recomendación y guía para la prevención del SMSL. Conclusiones: el SMSL posiblemente esté infrarreportado y asociado a otros diagnósticos, se recomienda que los profesionales de la salud insistan y alienten a las madres a que pongan al recién nacido a dormir en decúbito supino, con el fin de prevenir el SMSL.

Palabras clave: Muerte súbita; Recién nacido; Posición supina.

\section{Introdução}

A Síndrome da Morte Súbita do Lactente (SMSL) pode acometer crianças saudáveis, cujo óbito acredita-se ocorrer durante o sono. O lactente encontra-se em um processo de adaptação ao meio, amadurecendo seus sistemas fisiológicos. A posição mais indicada para o sono bem como os impactos que esta decisão tem para a síndrome são pauta recorrente em pesquisas e discussões sobre a orientação do decúbito durante o sono. A SMSL teve seu conceito definido por volta de 1969, quando crianças saudáveis menores de um ano faleciam subitamente. Diante do ocorrido a causa permanecia sem explicação, mesmo depois de uma minuciosa investigação (Chapur, et al., 2019).

A comunidade científica nacional percorreu um longo caminho para a compreensão dos mecanismos dos possíveis fatores de riscos associados à síndrome, incluindo o ambiente durante o sono. Pontua-se que o posicionamento na hora de dormir interfere de forma significativa no desenvolvimento da SMSL, principalmente por se tratar de uma “janela de prevenção”, sendo o posicionamento para dormir uma oportunidade de intervenção preemptiva para o profissional de saúde.

A SMSL não abrange somente uma causa e sua investigação demanda uma inspeção não só da saúde do lactente, mas da ambiência durante o sono (Castro, et al., 2016). Assim, pensando de modo multicausal, existem hipóteses que tentam esclarecer os fatores da morte súbita dentre os quais as condições que envolvem o ambiente na hora de dormir e uma gestação negligente em acompanhamento e autocuidado.

Observa-se que a SMSL, é identificada como a terceira maior causa de mortalidade infantil nos EUA. Desta sorte, houve uma campanha nacional de conscientização pública, "Back to Sleep”, com enfoque no posicionamento em decúbito dorsal do bebê durante o sono, para reduzir o risco da SMSL (Colson et. al, 2015). Como resultado houve redução da mortalidade pela metade, porém em 2014, ocorreram 3500 mortes infantis sugerindo asfixia, estrangulamento acidental ou causas mal definidas, identificadas como SMSL. Visto que os esforços da equipe de saúde pública não vêm declarando êxito na adoção do comportamento relacionado ao compartilhamento de camas e ao uso de camas macias. 
Enquanto isso no Brasil, a Sociedade Brasileira de Pediatria (SBP) afirma que não há dados oficiais sobre a prevalência desta síndrome no país. O centro de pesquisa Epidemiológicas da Universidade Federal de Pelotas (UFPel) e campanhas recentes divulgadas nos Estados Unidos e na Europa, divulgaram que posicionar o lactente para dormir de barriga para cima pode reduzir em $70 \%$ o risco da SMSL, e baseado nesta informação a Pastoral da Criança lançou uma campanha no dia 22 de junho de 2009, "Dormir de barriga para cima é mais seguro", o primeiro passo para adotar as novas diretrizes com relação ao sono infantil no Brasil.

Nesse contexto, insere-se o profissional enfermeiro possui um papel de extrema importância, visto que é responsável pelo acompanhamento do binômio materno-infantil e, ainda, tem como competência a realização de intervenções educativas e inovadoras com abordagem e apresentação adequada aos diversos contextos e arranjos familiares e sociais para que se compreenda os métodos de prevenção da SMSL. Destaca-se, que os cuidados para a prevenção da SMSL, são voltadas principalmente aos cuidadores dos lactentes que não sejam profissionais da saúde, sejam esses de origem familiar ou não (Dias, et al., 2018).

A elaboração deste artigo justifica-se pela necessidade de esclarecer uma melhor abordagem terapêutica para evitar o desenvolvimento desta síndrome, para o esclarecimento de dúvidas e correção/adequação de condutas. Com isso, demonstra-se sua relevância por se tratar de uma defunção abrupta de lactentes sem uma causa óbvia, pelo número relevante de óbitos infantis, falta ou desatualização de conhecimento por parte do cuidador e para adesão de medidas preventivas comprobatórias a serem alcançadas.

Diante disto, esta pesquisa tem por escopo identificar e orientar o posicionamento potencialmente redutor do acometimento da Síndrome da Morte Súbita do Lactente, com a finalidade de responder a seguinte questão norteadora: qual o posicionamento é mais adequado ao recém-nascido e lactente durante o sono para reduzir o acometimento da Síndrome da Morte Súbita do Lactente?

\section{Metodologia}

A Revisão Integrativa (RI) é uma ferramenta largamente utilizada para orientar a Prática Baseada em Evidências (PBE) no cuidado em saúde, com a finalidade de promover discussões entre os resultados e eficácia de abordagens terapêuticas publicadas em artigos científicos. Assim, esta RI sistematizou e comparou a eficácia de duas posições para colocar o recém-nascido e lactente durante o sono para prevenir a Síndrome da Morte Súbita do Lactente (SMSL): o decúbito dorsal ou o decúbito ventral. Esta pesquisa é coordenada por um protocolo que se sucedeu em seis fases, a partir do qual foi possível eleger a intervenção com êxito superior ao que tange ao número de óbitos por SMSL (Mendes, et al., 2008).

1. Identificação do tema e elaboração da questão de pesquisa: A problemática em questão alude ao impacto da prática do decúbito dorsal ou ventral durante o sono na prevalência da Síndrome da Morte Súbita do Lactente. Destaca-se que foram seguidas as recomendações do checklist com 27 itens do Statement for Reporting Systematic Reviews and Meta-Analyses of Study (PRISMA). Nesse sentido, utilizou-se a estratégia PICO para elaboração da pergunta norteadora. Essa estratégia representa um acrônimo para Paciente, Intervenção, Comparação e Outcomes. Outrossim, o P se referiu aos Lactentes, I ao posicionamento durante o sono, C apresenta as práticas dos decúbitos dorsal em detrimento do ventral, e O a Síndrome da Morte Súbita do Lactente. Qual posicionamento é adequado ao recém-nascido/lactante durante o sono para reduzir o acometimento da Síndrome da Morte Súbita do Lactente?

2. Amostragem ou pesquisa da literatura: Os descritores utilizados pertencem à lista de Descritores em Ciências da Saúde (DeCS), sendo eles: Morte súbita; neonato; recém-nascido; decúbito dorsal; decúbito ventral. 
Empregou-se a busca avançada com o uso do operador booleano "and" e "or", que permitiu acessar os artigos que possuem intersecção entre os diferentes descritores, por meio do portal de periódicos CAPES com acesso institucional irrestrito foram acessadas as bases de dados Scientific Electronic Library Online (Scielo), CINAHL, Literatura LatinoAmericana e do Caribe em Ciências da Saúde (LILACS), Embase e Science Direct e por meio da Biblioteca Virtual em Saúde (BVS) foram acessadas as bases Medline, SCOPUS MedCarib, Wholis e PAHO. Os casos de divergências quanto à inclusão de um estudo, foi consultado o pesquisador mais experiente.

3. Categorização dos estudos: Os escritos foram revisados de modo interdependente por cada autora e posteriormente sumarizados criando um banco de dados a partir do título; orientação de qual decúbito utilizar; autoria/ano; instituição sede ou local do estudo; características metodológicas do estudo; e avaliação do rigor metodológico a partir do Sistema GRADE (Grading of Recommendations Assessment, Development and Evaluation). Os estudos agrupam-se conforme o determinante da doença em três categorias: 1- baixa escolaridade/socioeconômicos, 2ausência ou fragilidade nas orientações durante o pré-natal, 3- capacitação profissional. Os critérios de inclusão foram artigos com disponibilidade na íntegra e gratuito, nos idiomas português, inglês ou espanhol, publicados no período de 2015 à fevereiro de 2020, que explicitassem em título, resumo e palavras-chave no que concerne a SMSL. Os critérios de exclusão foram os artigos não pertinentes, não disponíveis na integra, publicados fora do período, estudos realizados com animais, trabalhos apresentados em eventos, relato de experiencia e de caso, teses, dissertações e trabalhos de conclusão de curso.

4. Análise crítica dos estudos incluídos: foi avaliada a capacidade dos estudos de explicar a SMSL; contribuição da equipe de saúde na adoção do posicionamento do lactente durante o sono, qual posição foi a mais indicada e como se justifica.

5. Interpretação e discussão dos resultados: o conteúdo dos artigos foi apresentado e discutido à luz da literatura pertinente de acordo com a ordem apresentada na tabela dos resultados. Em seguida criou-se uma síntese com a conclusão a respeito de qual posicionamento é mais indicado. Por fim, apresentou-se recomendações para a Prática Baseada em Evidências.

6. Apresentação da revisão/síntese de conhecimento: Foi criada uma síntese explicando o que é a SMSL e o posicionamento mais indicado durante o sono a fim de evitá-la, reforçando o papel do enfermeiro enquanto educador. Foram construídos quadros, figuras e tabelas como recurso visual para a compreensão do leitor mediante a apresentação dos dados apurados.

\subsection{Avaliação das evidências}

A avaliação do nível de confiabilidade das evidências eleitas se deu através do sistema GRADE (Grading of Recommendations Assessment, Development and Evaluation), manual de graduação da qualidade da evidência e força de recomendações para tomada de decisão em saúde, que foi desenvolvido por um grupo colaborativo de pesquisadores que visa à criação de um sistema universal, transparente e sensível para a qualidade das evidências e a força das recomendações. É amplamente em pesquisas da área de saúde, na elaboração de recomendações com o paradigma Saúde Baseada em Evidências ilustrado na Tabela 1 abaixo: 
Tabela 1 - Níveis de evidência de acordo com o Sistema Grading of Recommendations Assessment, Development and Evaluation (GRADE) - Maceió, AL, 2020.

\begin{tabular}{|c|c|c|c|}
\hline NÍVEL & DEFINIÇÃO & IMPLICAÇÃO & FONTE DE INFORMAÇÃO \\
\hline ALTO & $\begin{array}{l}\text { Há forte confiança de } \\
\text { que o verdadeiro efeito } \\
\text { esteja próximo daquele } \\
\text { estimado. }\end{array}$ & $\begin{array}{l}\text { É improvável que } \\
\text { trabalhos adicionais } \\
\text { irão modificar a } \\
\text { confiança na estimativa } \\
\text { do efeito. }\end{array}$ & $\begin{array}{l}\text {-Ensaios clínicos } r \text { bem } \\
\text { delineados, com amostra } \\
\text { representativa. } \\
\text { casos, estudos observacionais } \\
\text { bem delineados, com achados } \\
\text { consistentes*. }\end{array}$ \\
\hline MODERADO & $\begin{array}{lr}\text { Há } & \text { confiança } \\
\text { moderada } & \text { no efeito } \\
\text { estimado. } & \end{array}$ & $\begin{array}{l}\text { Trabalhos futuros } \\
\text { poderão modificar a } \\
\text { confiança na estimativa } \\
\text { de efeito, podendo, } \\
\text { inclusive, modificar a } \\
\text { estimativa. }\end{array}$ & $\begin{array}{l}\text { - Ensaios clínicos com } \\
\text { limitações leves**. - Estudos } \\
\text { observacionais bem delineados, } \\
\text { com achados consistentes*. }\end{array}$ \\
\hline BAIXO & $\begin{array}{l}\text { A confiança no efeito é } \\
\text { limitada. }\end{array}$ & $\begin{array}{l}\text { Trabalhos futuros } \\
\text { provavelmente terão } \\
\text { um impacto importante } \\
\text { em nossa confiança na } \\
\text { estimativa de efeito. }\end{array}$ & $\begin{array}{lc}\text { - Ensaios } & \text { clínicos com } \\
\text { limitações } & \text { moderadas } * * \\
\text { Estudos } & \text { observacionais } \\
\text { comparativos: } & \text { coorte e caso- } \\
\text { controle. } & \end{array}$ \\
\hline
\end{tabular}

*Estudos de coorte sem limitações metodológicas, com achados consistentes apresentando tamanho de efeito grande e/ou gradiente dose resposta. **Limitações: vieses no delineamento do estudo, inconsistência nos resultados, desfechos substitutos ou validade externa comprometida. Fonte: Adaptação de GRADE working group pelos autores (2021).

\section{Resultados}

\subsection{Processo de exclusão/inclusão dos registros encontrados}

A partir da busca realizada foram encontrados, inicialmente, 16.147 registros. Destes, foram lidos os títulos, resumos e descritores, por quatro pesquisadoras, que se distribuíram nas bases de dados: Scientific Electronic Library Online (Scielo), CINAHL, Science Direct, Medline, Literatura Latino-Americana e do Caribe em Ciências da Saúde (LILACS), MedCarib, PAHO, SCOPUS, Wholis e Embase.

Seguindo o processo de identificação, seleção e inclusão dos artigos investigados descrito na Figura 1, dos 49 artigos identificados, 12 eram registros duplicados e foram removidos, 16 foram selecionados e dois foram excluídos, por se tratar de um artigo de revisão e inexistência da posição para dormir a fim de evitar a SMSL, respectivamente, 14 artigos se qualificaram para a análise. A principal língua de divulgação desses manuscritos foi o inglês (10 artigos), seguido dos artigos em português e espanhol (1 e 3, respectivamente). As produções analisadas foram publicadas em quatro bases de dados (Medline, Scopus, LILACS e Embase), sendo duas em periódicos de enfermagem. 
Figura 1 - Fluxograma de identificação, seleção e inclusão dos artigos investigados - Maceió, AL, 2021.

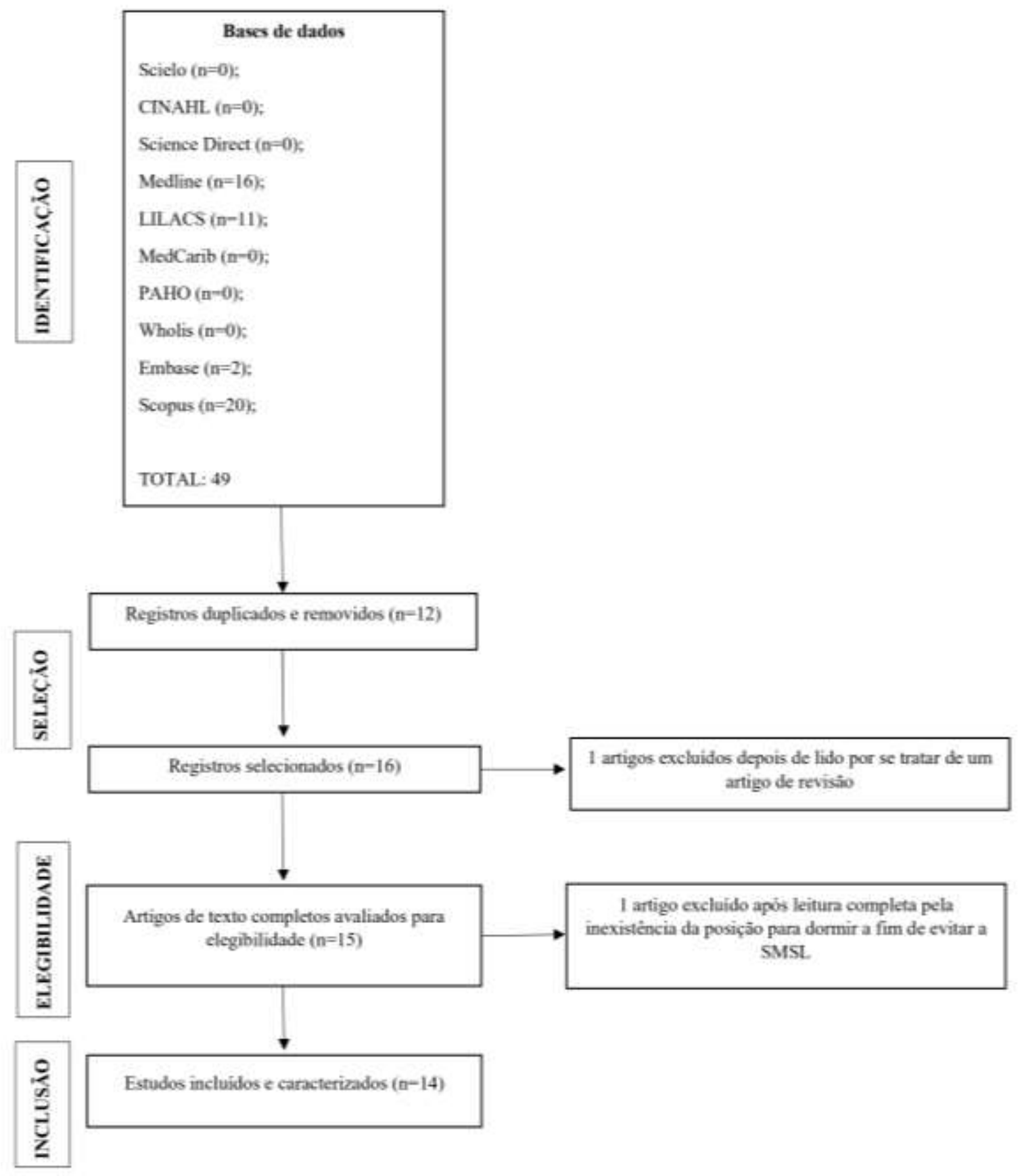

Fonte: Autores (2021).

\subsection{Caracterização das pesquisas}

Verificou-se que, das 14 pesquisas encontradas no período estudado, o maior número de publicações ocorreu no ano de 2016 (38\%), seguido dos anos de 2015 (23\%), 2017 e 2018 (15\%) e 2019 (8\%). As pesquisas sobre a SMSL se concentraram no continente norte americano (43\%) e sul-americano (36\%). A Tabela 2 representa uma sumarização dos artigos selecionados, onde foi avaliado o rigor metodológico a partir do Sistema GRADE (Grading of Recommendations Assessment, Development and Evaluation). 
Tabela 2 - Sumarização das pesquisas sobre as posições para colocar o neonato e lactente durante o sono para prevenir a Síndrome da Morte Súbita do Lactente (SMSL) - Maceió, AL, 2021.

\begin{tabular}{|c|c|c|c|c|c|c|}
\hline ID & Título & País & Autoria/ano & $\begin{array}{l}\text { Rigor } \\
\text { metodológic } \\
\text { o }\end{array}$ & $\begin{array}{l}\text { Características } \\
\text { metodológicas do } \\
\text { estudo }\end{array}$ & $\begin{array}{l}\text { Orientação } \\
\text { decúbito }\end{array}$ \\
\hline A1 & $\begin{array}{l}\text { Intenção das mães em } \\
\text { colocar o bebê para } \\
\text { dormir em decúbito } \\
\text { dorsal: um estudo de } \\
\text { base populacional }\end{array}$ & Brasil & $\begin{array}{l}\text { Cesar, JA, Acevedo, JD, } \\
\text { Kaczan, CR, Venzo, } \\
\text { JCP, Costa, LR \& Silva } \\
\text { LCM/2018 }\end{array}$ & Moderado & $\begin{array}{l}\text { Pesquisa } \\
\text { quantitativa } \\
\text { transversal } \\
\text { abordagem } \\
\text { descritiva }\end{array}$ & Decúbito dorsal \\
\hline A2 & $\begin{array}{l}\text { Morte infantil } \\
\text { inesperada. Análise de } \\
591 \text { casos }\end{array}$ & Uruguai & $\begin{array}{l}\text { Gutiérrez, } \\
\text { Rodríguez, }\end{array}$ & Alto & $\begin{array}{l}\text { Pesquisa } \\
\text { quantitativa } \\
\text { transversal } \\
\text { abordagem } \\
\text { explicativa }\end{array}$ & Decúbito ventral \\
\hline A3 & $\begin{array}{l}\text { Síndrome infantil de } \\
\text { morte súbita: o risco } \\
\text { enfrentado por } \\
\text { crianças em Bogotá, } \\
\text { DC, Colômbia }\end{array}$ & $\begin{array}{l}\text { Colômbi } \\
\text { a }\end{array}$ & $\begin{array}{l}\text { Latorre-Castro M } \quad \text { L, } \\
\text { Zambrano-Pérez C } \quad \& \\
\text { Carrascal-Gordillo } \\
\text { CF/2016 }\end{array}$ & Alto & $\begin{array}{l}\text { Pesquisa } \\
\text { quantitativa } \\
\text { transversal } \\
\text { abordagem } \\
\text { descritiva } \\
\end{array}$ & Decúbito dorsal \\
\hline A4 & $\begin{array}{l}\text { O efeito da melhoria } \\
\text { da qualidade da } \\
\text { enfermagem e } \\
\text { intervenções móveis } \\
\text { de saúde nas práticas } \\
\text { de sono infantil: um } \\
\text { ensaio clínico } \\
\text { randomizado }\end{array}$ & $\begin{array}{l}\text { Estados } \\
\text { Unidos }\end{array}$ & $\begin{array}{l}\text { Moon RY, Hauck FR, } \\
\text { Colson ER, Kellams } \\
\text { AL, Geller NL, Heeren } \\
\text { T, Kerr SM Drake EE, } \\
\text { Tanabe K, McClain M } \\
\text { \& Corwin MJ/2017 }\end{array}$ & Moderado & $\begin{array}{l}\text { Pesquisa } \\
\text { quantitativa } \\
\text { longitudinal } \\
\text { abordagem } \\
\text { exploratória }\end{array}$ & Decúbito dorsal \\
\hline A5 & $\begin{array}{l}\text { Epidemiologia das } \\
\text { alterações súbitas } \\
\text { infantis inesperadas } \\
\text { na Argentina: } \\
\text { tendência secular e } \\
\text { variação espacial. }\end{array}$ & $\begin{array}{l}\text { Argenti } \\
\text { na }\end{array}$ & $\begin{array}{l}\text { Chapur VF, Alfaro EL, } \\
\text { Bronberg R \& Dipierri } \\
\text { JE/2019 }\end{array}$ & Moderado & $\begin{array}{l}\text { Pesquisa } \\
\text { quantitativa } \\
\text { prospectiva com } \\
\text { abordagem } \\
\text { descritiva }\end{array}$ & Decúbito dorsal \\
\hline A6 & $\begin{array}{lcr}\text { Resposta } & \text { a } & \text { uma } \\
\text { questão nacional: indo } \\
\text { além de "voltar a } \\
\text { dormir" em Três } \\
\text { Hospitais }\end{array}$ & $\begin{array}{l}\text { Estados } \\
\text { Unidos }\end{array}$ & $\begin{array}{l}\text { Sleutel, MR., True, B., } \\
\text { Gustus, H., Baldwin, } \\
\text { K., \& Early, B/2018 }\end{array}$ & Moderado & $\begin{array}{l}\text { Pesquisa } \\
\text { quantitativa } \\
\text { longitudinal com } \\
\text { abordagem } \\
\text { exploratória } \\
\end{array}$ & Decúbito dorsal \\
\hline A7 & $\begin{array}{l}\text { Estudo randomizado } \\
\text { de um livro infantil } \\
\text { versus brochuras para } \\
\text { conhecimento e } \\
\text { adesão ao sono seguro } \\
\text { em uma população de } \\
\text { alto risco }\end{array}$ & $\begin{array}{l}\text { stados } \\
\text { Unidos }\end{array}$ & $\begin{array}{l}\text { Hutton, J. S., Gupta, R., } \\
\text { Gruber, R., Berndsen, } \\
\text { J., DeWitt, } \quad \text { T., } \\
\text { Ollberding, N. J., Van } \\
\begin{array}{l}\text { Ginkel, J. B., \& } \\
\text { Ammerman, R. T/2017 }\end{array}\end{array}$ & Alto & $\begin{array}{l}\text { Pesquisa } \\
\text { qualitativa } \\
\text { transversal com } \\
\text { abordagem } \\
\text { exploratória }\end{array}$ & Decúbito dorsal \\
\hline A8 & $\begin{array}{lr}\text { O potencial } & \text { da } \\
\text { economia } & \\
\text { comportamental para } \\
\text { promover } & \text { práticas } \\
\text { seguras de } & \text { sono } \\
\text { infantil }\end{array}$ & $\begin{array}{l}\text { Estados } \\
\text { Unidos }\end{array}$ & $\begin{array}{l}\text { Stevens, J. \& Kelleher, } \\
\text { KJ/2016 }\end{array}$ & Baixo & $\begin{array}{l}\text { Pesquisa } \\
\text { qualitativa com } \\
\text { abordagem } \\
\text { exploratória }\end{array}$ & Decúbito dorsal \\
\hline A9 & $\begin{array}{l}\text { Fatores sociais } \mathrm{e} \\
\text { comportamentais na } \\
\text { morte inesperada não } \\
\text { suspeita na } \\
\text { infância; experiência } \\
\text { da investigação índigo } \\
\text { do projeto da polícia } \\
\text { metropolitana }\end{array}$ & $\begin{array}{l}\text { Reino } \\
\text { Unido }\end{array}$ & $\begin{array}{l}\text { Bamber AR, Kiho L, } \\
\text { Upton S, Orchard M \& } \\
\text { Sebire NJ/2016 }\end{array}$ & Moderado & $\begin{array}{l}\text { Pesquisa } \\
\text { quantitativa } \\
\text { retrospectivos } \\
\text { com abordagem } \\
\text { exploratória }\end{array}$ & Decúbito dorsal \\
\hline A10 & $\begin{array}{l}\text { A posição semi-prona } \\
\text { pode influenciar a } \\
\text { variabilidade na } \\
\text { frequência respiratória } \\
\text { de bebês prematuros }\end{array}$ & China & $\begin{array}{l}\text { Yin T, Yuh Y, Liaw J, } \\
\text { Chen Y \& } \text { Wang } \\
\text { K/2016 }\end{array}$ & Alto & $\begin{array}{l}\text { Pesquisa } \\
\text { quantitativa com } \\
\text { abordagem } \\
\text { descritiva }\end{array}$ & Decúbito ventral \\
\hline
\end{tabular}




\begin{tabular}{|c|c|c|c|c|c|c|}
\hline & usando CPAP nasal. & & & & & \\
\hline A11 & 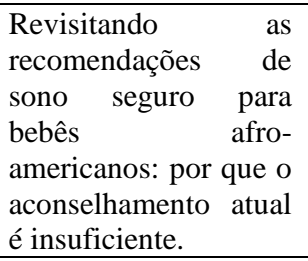 & $\begin{array}{l}\text { Estados } \\
\text { Unidos }\end{array}$ & $\begin{array}{lr}\text { Gaydos LM, Blake } & \text { SC, } \\
\text { Gazmararian } & \text { JA, } \\
\text { Woodruff } & \text { W, } \\
\text { Thompson WW } & \text { \& } \\
\text { Dalmita SG/2015 } & \end{array}$ & Moderado & $\begin{array}{ll}\text { Pesquisa } & \\
\text { qualitativa } & \text { com } \\
\text { abordagem } & \\
\text { explicativa } & \end{array}$ & Decúbito dorsal \\
\hline A12 & $\begin{array}{l}\text { A autópsia molecular } \\
\text { em uma coorte de } \\
\text { bebês } \quad \text { morreu } \\
\text { repentinamente em } \\
\text { repouso }\end{array}$ & Uruguai & $\begin{array}{lr}\text { Campuzano } & \mathrm{O}, \\
\text { Beltramo P, Fernandez } \\
\text { A, et al/2018 }\end{array}$ & Alto & $\begin{array}{l}\text { Pesquisa caso } \\
\text { controle descritivo } \\
\text { quantitativa com } \\
\text { abordagem } \\
\text { experimental }\end{array}$ & Decúbito dorsal \\
\hline A13 & $\begin{array}{lr}\text { Morte } & \text { súbita } \\
\text { inexplicável } & \text { na } \\
\text { infância: } & \text { uma } \\
\text { comparação de } & \text { casos } \\
\text { com e sem } & \text { histórico } \\
\text { de crises } & \text { febris. } \\
\text { Epilepsia } & \\
\end{array}$ & $\begin{array}{l}\text { Estados } \\
\text { Unidos }\end{array}$ & $\begin{array}{l}\text { Hesdorffer } \quad \text { DC, } \\
\text { Crandall LA, Friedman } \\
\text { D \& Devinsky O/2015 }\end{array}$ & Moderado & $\begin{array}{l}\text { Pesquisa } \\
\text { quantitativa com } \\
\text { abordagem } \\
\text { descritiva }\end{array}$ & Decúbito dorsal \\
\hline A14 & $\begin{array}{l}\text { Conhecimento } \mathrm{e} \\
\text { crenças de pais e } \\
\text { apoiadores indianos } \\
\text { afro-americanos e } \\
\text { americanos sobre o } \\
\text { sono seguro do bebê. }\end{array}$ & $\begin{array}{l}\text { Estados } \\
\text { Unidos }\end{array}$ & $\begin{array}{l}\text { Herman SA, Mary L \& } \\
\text { Rachel Y/2015 }\end{array}$ & Moderado & $\begin{array}{l}\text { Pesquisa de } \\
\text { levantamento de } \\
\text { dados explicativa } \\
\text { com abordagem } \\
\text { qualitativa quase } \\
\text { experimental }\end{array}$ & Decúbito dorsal \\
\hline
\end{tabular}

Fonte: Autores (2021).

Conforme a Tabela 3, os artigos que averiguaram as posições para colocar o recém-nascido e lactente durante o a SMSL no âmbito da enfermagem, desconsiderando os não especificados, foram realizados em igual distribuição para hospitais, domicílios, e através de meios eletrônicos, bem como utilizando bases de dados secundárias.

Tabela 3 - Locais onde foram realizadas as pesquisas sobre a posições para colocar o neonato e lactente durante o sono para prevenir a Síndrome da Morte Súbita do Lactente (SMSL) - Maceió, AL, 2021.

\begin{tabular}{lcc}
\hline Locais dos estudos & Artigos N=14 & Frequência Relativa (FR)\% \\
\hline Não especifico & 5 & 36,0 \\
\hline Hospital & 2 & 14,0 \\
\hline Domicílio & 2 & 14,0 \\
\hline Meios eletrônicos & 2 & 14,0 \\
\hline Bases de dados epidemiológicos & 2 & 14,0 \\
\hline Maternidade & 1 & 7,0 \\
\hline TOTAL & $\mathbf{1 4}$ & $\mathbf{1 0 0}$ \\
\hline
\end{tabular}

Fonte: Autores (2021).

Ressalta-se que a maioria das pesquisas foram realizadas em hospitais (14\%), domicilio (14\%), por meios eletrônicos (14\%), bases de dados epidemiológicos (14\%), em maternidades (7\%) e em locais não específicos (36\%).

A Tabela 4 apresenta um panorama referente ao tipo de metodologia empregada nas investigações. Os tipos de estudos, objetivo, abordagem e delineamento dos artigos sobre as posições para colocar o neonato e lactente durante o sono para prevenir a SMSL. 
Tabela 4 - Panorama dos métodos empregados nos artigos sobre as posições para colocar o neonato e lactente durante o sono para prevenir a Síndrome da Morte Súbita do Lactente (SMSL) - Maceió, AL, 2021.

\begin{tabular}{lll}
\hline Tipo de estudo & Artigos N=14 & FR\% \\
\hline Caso Controle & 7 & 50,0 \\
\hline Levantamento & 5 & 36,0 \\
\hline Estudo de caso & 1 & 7,0 \\
\hline Coorte & 1 & 7,0 \\
\hline Objetivo & Artigos & FR\% \\
\hline Descritivo & 6 & 43,0 \\
\hline Exploratório & 5 & 36,0 \\
\hline Explicativo & 31,0 \\
\hline Abordagem & $\mathbf{F R \%}$ \\
\hline Qualitativa & $\mathbf{A r t i g o s}$ & 29,0 \\
\hline Quantitativa & 4 & 71,0 \\
\hline Delineamento & 10 & $\mathbf{F R \%}$ \\
\hline Experimental & $\mathbf{A r t i g o s}$ & 43,0 \\
\hline Quase experimental & 6 & 36,0 \\
\hline Não experimental & 5 & 21,0 \\
\hline Total & 3 & $\mathbf{1 0 0 \%}$ \\
\hline
\end{tabular}

Fonte: Autores (2021).

Os artigos, em sua maioria, são estudos de caso controle (50\%), seguidos dos artigos de levantamento de dados, estudo de caso e coorte, respectivamente $(36 \%, 7 \%, 7 \%)$. Com os objetivos predominantemente descritivos (43\%), seguidos dos exploratórios e explicativos, respectivamente (36\% e 21\%). Quanto a abordagem, corresponde a quantitativa (71\%) e qualitativa (29\%). Em relação ao delineamento, encontrou-se estudos experimentais (43\%), quase experimentais (39\%) e não experimental (21\%). A tabela 5 analisa os mecanismos de lesão associados a SMSL.

Tabela 5 - Mecanismos de lesão associados a Síndrome da Morte Súbita do Lactente (SMSL) - Maceió, AL, 2021.

\begin{tabular}{lcl}
\hline Mecanismos de lesão & Artigos N=14 & FR \% \\
\hline Uso de colecho/Co-sleeping (dormir com o bebê) & 7 & 21,0 \\
\hline Exposição ao tabaco & 6 & 18,0 \\
\hline Objetos macios na cama & 6 & 18,0 \\
\hline Decúbito lateral & 5 & 15,0 \\
\hline Uso de drogas e álcool durante a gestação & 4 & 12,0 \\
\hline Uso de cobertores & 4 & 12,0 \\
\hline TOTAL & $\mathbf{1 4}$ & $\mathbf{1 0 0}$ \\
\hline
\end{tabular}

Fonte: Autores (2021).

Os principais mecanismos de lesão descritos na literatura relacionados a SMSL e foram identificados desde mecanismos físicos ou ambientais como a presença de objetos (18\%), cobertores (12\%), exposição ao tabaco (18\%), fatores comportamentais como o decúbito lateral (15\%), o ato de dormir com o bebê (21\%) e, ainda, o impacto de álcool e outras drogas durante a gestação (12\%), como exposto na Tabela 5.

\section{Discussão}

Nossos achados demonstraram a predominância do decúbito dorsal como medida de recomendação e orientação da maioria dos estudos de caso-controle descritivos com síntese quantitativa de delineamentos experimentais, estes 
foram realizados nos mais diversos ambientes. Dessa forma, dividimos em categorias para uma melhor compreensão da indicação na literatura existente. Na primeira categoria enquadram-se as causas de óbito relacionadas ao decúbito dorsal, na segunda ao decúbito ventral e na terceira ao decúbito lateral.

\subsection{Categoria 1: Acometimento da Síndrome da Morte súbita do Lactente associada ao decúbito dorsal}

Recomenda-se como como cuidados em relação ao posicionamento dos lactentes para dormir: posição supina, em superfícies firmes, no quarto dos pais e em cama/berço próprio, não praticar o co-leito, evitar sobreaquecimento e exposição à fumaça do tabaco, além de estimular a amamentação (Herman, et al., 2015). Aliado a isso, para a posição mais adequada para os bebês dormirem seria em decúbito dorsal (deitado de costas) até o primeiro ano de vida. Considera-se assim, que essa posição não aumenta o risco de asfixia e aspiração nos lactentes, mesmo aquelas com refluxo gastroesofágico (Lazzaroto, et al., 2019).

Além disso, o estudo de base populacional realizado em Rio Grande (RS) mostrou que quanto maior a paridade, menor razão de prevalência para intenção de a mãe colocar o bebê para dormir em decúbito dorsal. depreende-se desta forma que quanto maior a idade, em geral, maior a paridade e, portanto, maior a experiência para com cuidados direcionados ao bebê. E, dentre as mães com nível socioeconômico mais baixo, atendidas nas UBS, houve maior resistência à adesão ao decúbito dorsal. Dessa forma, conclui-se que a maior predisposição em colocar o bebê para dormir de barriga para cima possivelmente decorre do melhor nível de escolaridade das mães consultadas (Cesar, et al., 2018).

Investigado o conhecimento materno a respeito da SMSL, apenas $15,8 \%$ das mães tinham ouvido falar da síndrome e destas, 29,4\% citavam como medida preventiva o decúbito dorsal para o lactente dormir. Acrescido a isso, sugestões de realizar campanhas de incentivo à posição dorsal para o sono infantil contribuem para a adesão a essa prática (Bezerra, et al., 2015).

Destaca-se a importância da realização de ensaios clínicos randomizados para melhorar as práticas de sono seguro infantis. Em um ensaio de intervenções educacionais com provedores de cuidados infantis dos EUA melhorou a colocação relatada da posição supina de sono de $65,0 \%$ a $87,8 \%$ e observou a colocação em posição supina de sono de 51,0\% para 62,1\% (Moon, et al., 2017). A educação individual com mães brasileiras mostrou melhora na adesão de taxas de posição supina para dormir 2,2 vezes 3 meses após a intervenção (Issler, et al., 2009).

Acrescido a isso, um estudo longitudinal quase experimental realizado em três hospitais nos Estados Unidos, demonstrou que a aplicabilidade do mnemônico ABC’S, o qual inclúa: A (Sozinho) - O bebê dorme sozinho, sem objetos macios na cama; B (Costas/Cobertores) - O bebê dorme de costas. Não use cobertores para enfaixar; C (Berço/Legal) - O bebê dorme no berço. O bebê deve ser legal (normal/confortável, temperatura ambiente, bebê não excessivamente vestido); S (Adormecido/Fumador) - O bebê não deve ser segurado por um adulto adormecido. Evite a exposição de crianças ao fumo. Voltado para o ensino dos pais como ferramenta educacional atualizada melhorou o conhecimento e as práticas dos enfermeiros e pais sobre fatores de segurança e condições do sono infantil (Sleutel, et al., 2018).

Demonstra-se que a relação entre escolaridade e presença às consultas de pré-natal de mulheres, dentre as 2.395 (97,2\% do total) mães entrevistadas, $20,5 \%$ manifestaram intenção de colocar o bebê para dormir de barriga para cima. Esta intenção variou de $11 \%$, entre mães com três ou mais filhos, a 35\%, entre aquelas com 12 anos ou mais de escolaridade. Cerca de $80 \%$ realizaram seis ou mais consultas de pré-natal e 20,5\% disseram ter a intenção de colocar o filho recém-nascido para dormir de barriga para cima (posição supina) (Cesar, et al., 2018). 


\subsection{Categoria 2: Acometimento da Síndrome da Morte súbita do Lactente associada ao decúbito ventral}

A posição ventral (abdome para baixo) é considerada de maior risco para a Síndrome da Morte Súbita do Lactente, e assim deve ser evitada pela maioria das mães, para desse modo contribuir para a baixa prevalência da SMSL. Dessa forma, quando se coloca uma criança (0-6 meses) na posição ventral, a mesma não tem controle do tronco e nem faz extensão do pescoço, o que pode levar a morte, seja ela por asfixia ou por tal Síndrome (Lazzaroto, et al., 2019). Nesse contexto, salienta-se que a posição ventral deve ser considerada apenas em casos de anormalidades e distúrbios de vias aéreas superiores, nos quais o risco de morte por refluxo gastroesofágico extrapole o risco de SMSL (Oliveira, et al., 2020). Em uma análise de 591 casos de SMSL, observou-se que $72 \%$ dos casos estavam associados ao decúbito ventral junto a fatores de risco com: anomalia cardíaca, diarreia com desidratação, sufocação acidental e infecção respiratória (Gutiérrez, et al,. 2017).

Efeitos relacionados à posição mostram diferenças significativas na variabilidade da Frequência RespiratóriaFR (Saturação) demonstrando que os valores de FR são significativamente menores na posição Semiprona, a posição lateral foi associada ao aumento do FR (Stevens, et al., 2016). Nesse contexto, aponta-se que existe uma proporção considerável de mortes associadas a fatores de risco como co-leito, sendo tal comportamento associado a fatores sociais, incluindo questões de acomodação (Bamber, et al., 2016).

A Academia Americana de Pediatria passou a recomendar que crianças fossem colocados na posição em decúbito dorsal, de preferência em camas firmes e não compartilhadas, e essa recomendação é tentar manter o sono seguro de crianças, a questão cultural de como os pais colocam seus filhos para dormir (Gaydos et al., 2015). As crenças Afro-americanas e indígenas à prática do sono-seguro, mostraram-se semelhantes quanto a preocupações com conforto e segurança infantil. E muitas vezes, se sobressaem às recomendações de especialistas, a exemplo da crença de que uma mãe sabe instintivamente onde seu bebê está quando dorme na cama com a mesma, acordando assim, antes que o bebê esteja em perigo. Outra recorrente consistiu na escolha posição lateral como melhor alternativa para crianças que viessem a vomitar, eles não engasgariam, essas crenças podem influenciar diretamente no bem estar da criança ao leito (Yin, et al., 2016).

Existem poucas evidências que confirmam que a análise dos riscos aumenta com a influência de fatores térmicos, estimulando a criança para a posição decúbito ventral. Em uma série de casos foi relatado que $25-40 \%$ das crianças encontradas mortas tinham suas cabeças e faces cobertas por roupas de cama, o que causa estresse térmico ou sufocação por inalação de gases expirados (Mitchell, et al., 2006). As taxas de co-sono ficam em cerca de $40 \%$ dos casos relacionados para acometimento das crianças, além do fato deles partilharem cama com adultos o que colabora para a possibilidade da partilha da cama ser um fator que pode influenciar na segurança do sono seguro (Bamber, et al., 2016).

\subsection{Categoria 3: Acometimento da Síndrome da Morte súbita do Lactente associada ao decúbito lateral}

O decúbito lateral não é seguro, nem aconselhado (Oliveira, et al., 2020). Esta posição mostrou-se ser a preferencial para o sono dos lactentes, porém esta também é considerada uma posição de risco, visto que a criança tem maior facilidade em se colocar na posição de decúbito ventral (Lazzaroto, et al., 2019).

Ademais, um estudo realizado em Bariloche (2008), demonstrou que num total de 10 casos, $70 \%$ das crianças dormiam de lado. Do total de recomendações recebidas de profissionais da saúde, $61 \%$ foram instruídas a colocar o bebê de lado. O principal motivo para recomendar essa posição foi a prevenção do sufocamento ou engasgo (56\%) e aspiração ou broncoaspiração (20\%) (Chapur, et al., 2019).

É notória a incidência da SMSL em estratos socioeconômicos baixos, do que em estratos socioeconômicos altos, concluindo-se que o risco aumenta na população mais pobre (Latorre-Castro, et al., 2016). De forma analítica, a 
utilização de livros infantis para prevenção da SMSL demonstra adesão e conhecimento dos cuidadores sobre o sono seguro (Hutton, et al., 2017).

A SMSL é uma condição multifatorial em que diversos fatores predispõem a morte do lactente (Campuzano, 2018). Existem paralelos sobre a morte de bebês com Epilepsia em relação a posição de prona, em especial, mortes não testemunhadas, durante o sono, com predominância no sexo masculino, sugerindo a possibilidade de a morte ser associada à Febre Terminal que aumenta os riscos do paciente convulsionar, elevando o potencial de letalidade (Hesdorffer, et al., 2015).

Nesse contexto, insere-se a enfermagem e seu papel orientador de pais, mães e cuidadores em geral, visto que estudos apontam que mesmo os pais que realizam a prona do bebê para o sono adequado e seguro desconhecem as justificativas para tal (Herman, et al., 2015). Além do processo de desconhecimento, a enfermagem também deverá atuar na educação em saúde, unindo saberes do senso comum ao científico com a finalidade de aumentar a adesão terapêutica às indicações de sono seguro, dada a assistência contínua deste profissional (Ramos, et.al., 2018).

\section{Conclusão}

Constatou-se a partir da literatura consultada que dentre os posicionamentos para dormir o mais seguro é o decúbito dorsal, com o bebê em posição supina, em superfícies firmes e sem a presença de objetos ou de outros indivíduos no leito. Cabe fazer a ressalva de que muitos estudiosos sugerem o decúbito lateral como uma importante medida de prevenção ao engasgo e asfixia ou o decúbito ventral em crianças com distúrbios de vias aéreas superiores ou anormalidades gastroesofágicas.

Assim, recomenda-se para os profissionais de saúde repassarem, insistirem, incentivarem as mães a colocar o recém-nascido para dormir em uma posição segura. Isto porque se trata de uma medida simples, de baixo custo e de fácil aplicação com resultado comprovadamente eficaz. Em seu papel de educador, o enfermeiro tem como objetivo elementar prevenir e minimizar agravos, devendo, portanto, estabelecer estratégias de intervenção para promoção do sono saudável e seguro. A educação em saúde constitui uma estratégia capaz de favorecer a compreensão dos responsáveis pelas crianças acerca da SMSL.

\section{Referências}

American Academy of Pediatrics (2011). SMSL e outras mortes de bebês relacionadas ao sono: expansão das recomendações para um ambiente seguro para o sono infantil. Pediatria, 128 (5): 1-27. 10.1542 / peds.2011-2284

Bamber, A. R., et al (2016). Fatores sociais e comportamentais na morte inesperada não suspeita na infância, experiência da investigação índigo do projeto da polícia metropolitana. BMC Pediatr, 16 (1): 1-7. 10.1007/s10995-016-2163-1

Brasil. Ministério da Saúde (2014). Diretrizes Metodológicas Sistema GRADE - manual de graduação da qualidade da evidência e força de recomendação para tomada de decisão em saúde. Ministério da saúde. Secretaria de Ciência, Tecnologia e Insumos Estratégicos Departamento de Ciência e Tecnologia. https://pesquisa.bvsalud.org/bvsms/resource/pt/mis-37111

Brasil, P. R. C., \& Santos, A. M. (2019). Desafios às ações educativas das Equipes de Saúde Bucal na Atenção Primária à Saúde: táticas, saberes e técnicas. Physis: Revista de Saúde Coletiva [online], 28(4), e280414. 10.1590/S0103-73312018280414

Bezerra, M. A. L., et al (2015). Fatores associados ao conhecimento das mães sobre a Síndrome da Morte Súbita do Lactente. Esc. Anna Nery, 192, 303-309. 10.5935/1414-8145.20150041

Cesar, J. A., et al (2018). Intenção das mães em colocar o bebê para dormir em decúbito dorsal: um estudo de base populacional. Ciênc. saúde coletiva , 23 (2): 501-508. 10.1590/1413-81232018232.20732015

Chapur V. F, et al (2019). Epidemiologia das alterações súbitas infantis inesperadas na Argentina: tendência secular e variação espacial. Arco. argento. pediatr, 117 (3): 164-170. 10.5546/aap.2019.164.

Dias, E., et al (2018). Ações do enfermeiro no pré-natal e a importância atribuída pelas gestantes. Revista Sustinere, 6(1), 52 - 62. 10.12957/sustinere.2018.31722 
Gaydos L. M., et al (2015). Revisitando as recomendações de sono seguro para bebês afro-americanos: por que o aconselhamento atual é insuficiente. Diário de saúde materno infantil. 19 (3): 496-503. 10.12957/sustinere.2018.31722

Geib L. T., \& Nunes M. L. (2006). A incidência da síndrome de morte súbita em uma coorte de bebês. Jornal de Pediatria, 82(1), 21-26. 0021$7557 / 06 / 82-01 / 21$

Gutiérrez C., et al (2017). Muerte inesperada del lactante: Análisis de 591 casos. Archivos de Pediatría del Uruguay, 88(1), 12-18. http://www.scielo.edu.uy/scielo.php?script=sci_arttext\&pid=S1688-12492017000100004

Herman S. A., Mary L., \& Rachel Y. (2015). Conhecimento e crenças de pais e apoiadores indianos afro-americanos e americanos sobre o sono seguro do bebê. J Saúde Comunitária, 40 (1): 12-9. 10.1007/s10900-014-9886-y

Hesdorffer, D. C., et al (2015). Morte súbita inexplicada na infância: uma comparação de casos com e sem história de convulsão febril. Epilepsia, 56 (8), 1294-1300. doi 10.1016/j.jped.2015.08.003

Hutton, J. S., et al (2017). Teste aleatório de um livro infantil versus brochuras para conhecimento e adesão ao sono seguro em uma população de alto risco. Pediatria acadêmica, 17 (8), 879-886. 10.1016/ j.acap.2017.04.018

Instituto Brasileiro de Geografia e Estatística (2016). Diretoria de Pesquisas, Coordenação de Trabalho e Rendimento, Pesquisa Nacional por Amostra de Domicílios Contínua. https://www.ibge.gov.br/estatisticas/multidominio/condicoes-de-vida-desigualdade-e-pobreza/17270-pnad-continua.html

Issler R. M., Marostica P. J., \& Giugliani E. R (2009). Posição do bebê durante o sono: ensaio clínico randomizado de intervenção educativa na maternidade de Porto Alegre, Brasil. Nascimento, 36 (2): 115-121. 10.1111 / j.1523-536X.2009.00308.x

Latorre-Castro M. L., Zambrano-Pérez C., \& Carrascal-Gordillo C. F. (2016). Síndrome infantil de morte súbita: o que afrontam os lactantes em Bogotá, D.C., Colômbia. rev.fac.med, 64 (4): 665-670. 10.15446/revfacmed.v64n4.54801

Lazzaroto A. B., et al (2019). O uso da chupeta na prevenção da síndrome de morte súbita do lactente. Odontol. Clín.-Cient., Recife, $18(2) 87$ - 92. 10.1016/j.jped.2018.03.002

Martins M. E. P., et al (2018). Síndrome da Morte Súbita Infantil (SMSI): aspectos acerca das principais causas e as formas de prevenção. Revista Multidisciplinar e de psicologia, 12, 1981-1179. https://www.msdmanuals.com/pt-br/casa/problemas-de-sa\%C3\%BAde-infantil/dist\%C3\%BArbiosde-natureza-variada-em-beb\%C3\%AAs-e-crian\%C3\%A7as-pequenas/s\%C3\%ADndrome-de-morte-s\%C3\%BAbita-infantil-smsi

Mendes K. D. S., Silveira R. C. C P. \& Galvão C. M. (2009). Revisão integrativa: método de pesquisa para a incorporação de evidências na saúde e na enfermagem. Texto \& Contexto - Enfermagem, (17), 758-764. 10.1590/S0104-07072008000400018

Mitchell E. A., Blair P. S., \& L’hoir M. P (2006). A chupeta deve ser recomendada para prevenir a síndrome da morte súbita infantil? Academia Americana de Pediatria, (117), 1755-1758. 10.15600/2238-1236/fol.v24n1p51-57

Moon R. Y, et al (MJ (2017). O efeito da melhoria da qualidade da enfermagem e intervenções móveis de saúde nas práticas de sono infantil: um ensaio clínico randomizado. JAMA. 318 (4): 351-359. 10.1590/0034-7167-2016-0480

Oliveira A. M F, et al (2020). Fatores de risco e de proteção para a síndrome da morte súbita do lactente. Rev. Bras. Enferm. 10.1590/0034-7167-20190458

Ramos C. F. V, et al (2018). Práticas educativas: pesquisa-ação com enfermeiros da Estratégia de Saúde da Família. Rev. Bras. Enferm, 713 ): 1144 1151. 10.1590/0034-7167-2017-0284

Sleutel, M. R., et al (2018). Resposta a um problema nacional: indo além de "voltar a dormir" em três hospitais. Jornal de enfermagem pediátrica, 43, 16-22. 10.1016/j.pedn.2018.07.013

Stevens, J. \& Kelleher, K. J (2016). O potencial da economia comportamental para promover práticas seguras de sono infantil. Diário de Saúde Materno-Infantil . 21 (2) 229-233. 10.1590/2317-1782/20162014231

Yin, T., et al (2016). A posição semi-prona pode influenciar a variabilidade na frequência respiratória de bebês prematuros usando CPAP nasal. Jornal de enfermagem pediátrica, 31 (2), e167 - e174. 10.1016 / j.pedn.2015.10.014 